\title{
Resection of posterior clinoid meningioma through retrosigmoid approach: concepts and nuances
}

\author{
Anil Nanda, MD, MPH, Devi Prasad Patra, MD, MCH, Amey Savardekar, MD, MCH, \\ Tanmoy K. Maiti, MD, MCH, and Piyush Kalakoti, MD \\ Department of Neurosurgery, LSU Health Science Center, Shreveport, Louisiana

\begin{abstract}
Meningiomas arising from the posterior clinoid process pose a great surgical challenge because of their location and propensity to cause critical neurovascular compression. The authors' patient was a 66-year-old female who had a large posterior clinoid meningioma with significant brainstem compression that was operated on through the retrosigmoid approach. This 3D surgical video emphasizes the various technical concepts that are important to preserving compressed neural and vascular structures during the surgery. It would also be interesting to note the extent of visualization around the posterior clinoid region gained through a retrosigmoid corridor.
\end{abstract}

The video can be found here: https://youtu.be/CBmT_Oov0YA.

KEYWORDS posterior clinoid; meningioma; surgery; operative; video; retrosigmoid 